\title{
Hobnail variant of papillary thyroid carcinoma: a systematic review and meta-analysis
}

\author{
Lane B. Donaldson ${ }^{1} \cdot$ Flora Yan ${ }^{1} \cdot$ Patrick F. Morgan ${ }^{1} \cdot$ John M. Kaczmar ${ }^{2} \cdot$ Jyotika K. Fernandes $^{3}$. \\ Shaun A. Nguyen ${ }^{1} \cdot$ Rachel L. Jester $^{4} \cdot$ Terry A. Day $^{1}$
}

Received: 9 June 2020 / Accepted: 23 September 2020 / Published online: 6 October 2020

(c) Springer Science+Business Media, LLC, part of Springer Nature 2020

\begin{abstract}
Objective The hobnail variant of papillary thyroid carcinoma (HVPTC) has emerged as a rare and aggressive variant of papillary thyroid carcinoma (PTC). We aim to determine the prevalence and clinicopathologic factors of HVPTC.

Methods A systematic review of the literature for studies examining HVPTC was performed. Four databases (PubMed, Scopus, OVID, Cochrane library) were queried from inception of databases through March 20th, 2020.

Results Sixteen studies with 124 cases of HVPTC were included. The mean age for all patients was 52.3 years. HVPTC had a prevalence of $1.08 \%$ out of all PTC cases, with a mean tumor size of $3.1 \mathrm{~cm}$. In $62 \%$ and $50 \%$ of cases, lymphovascular invasion and extrathyroidal extension were present, respectively. Follow-up data, with a mean of 49.9 months, revealed a $66 \%$ rate of lymph node metastasis and $23 \%$ rate of distant metastasis. Tumors with $\geq 30 \%$ hobnail morphology had a 2.6-fold increased odds of developing lymph node metastasis compared with $<30 \%$ hobnail morphology, however did not differ in rates of distant metastasis. Patients $\geq 55$ years old had a 4.5 -fold increased odds of distant metastasis and a 4.7 -fold increased odds of lymphovascular invasion over patients $<55$.

Conclusions High rates of locoregional and distant disease as well as high-risk pathological factors reveal the aggressive nature of HVPTC. Diagnostic criteria regarding percentage of hobnail morphology requires further refinement. Further studies are warranted in order to better understand how recognition of this high-risk variant impacts clinical treatment.
\end{abstract}

Keywords Papillary thyroid cancer $\cdot$ Systematic review $\cdot$ Hobnail variant papillary thyroid cancer $\cdot$ Thyroid

\section{Introduction}

Supplementary information The online version of this article (https:// doi.org/10.1007/s12020-020-02505-z) contains supplementary material, which is available to authorized users.

Terry A. Day

headneck@musc.edu

1 Head and Neck Tumor Center, Department of Otolaryngology Head and Neck Surgery, Medical University of South Carolina, Charleston, SC 29425, USA

2 Head and Neck Tumor Center, Hollings Cancer Center, Department of Hematology/Oncology, Medical University of South Carolina, Charleston, SC 29425, USA

3 Division of Endocrinology, Diabetes, and Medical Genetics, College of Medicine, Medical University of South Carolina, Charleston, SC 29425, USA

4 Department of Pathology \& Laboratory Medicine, Medical University of South Carolina, Charleston, SC 29425, USA
Thyroid cancer is the most common endocrine malignancy with an incidence of $2.9 \%$ in the United States for the year 2018 alone [1]. There are multiple types of thyroid cancer with papillary thyroid carcinoma (PTC) being the most common, accounting for $\sim 80 \%$ of all cases [2, 3]. In general, welldifferentiated PTC is regarded as a fairly indolent tumor with long-term survival rates $>95 \%$ [4], however there are certain variants of PTC that are more aggressive in nature with lessfavorable disease-free survival and overall survival. These variants differ in histology, cytology, molecular markers, treatment strategies, and outcomes [5]. The hobnail variant of papillary thyroid carcinoma (HVPTC) has been recently identified as an aggressive variant of PTC, whose histological features were first described by Kakudo et al. in 2004 and further elaborated by Motosugi et al. in 2009 [6, 7]. A case series of eight patients from the Mayo Clinic further described HVPTC and found this variant to be associated with more aggressive behavior including increased radioactive iodine 
(RAI) refractoriness, rate of distant metastasis, and mortality when compared with classical PTC [8]. Additional studies have furthered our understanding of the clinicopathologic characteristics and prognostic implications of HVPTC.

Histologically, Kakudo et al. [6] initially noted an elevated nuclear position (described as analogous to a hobnail appearance) and proposed this loss of cellular polarity as a characteristic of poor cellular differentiation. Asioli et al. [8] refined the histopathological diagnosis of HVPTC and described three diagnostic criteria: (1) nonsolid type of PTC, (2) $\leq 10 \%$ of the tumor with tall/columnar or diffuse sclerosing features, and (3) loss of polarity/cohesiveness with hobnail features in $\geq 30 \%$ of the tumor cells. In addition, subsequent authors also identified those with hobnail features present in $10-30 \%$ of tumors cells demonstrated worse outcomes, indicating PTC with $<30 \%$ hobnail features are still aggressive [9]. Along with the hobnail appearance of cells, HVPTC demonstrates abundant cytoplasm with a higher nuclearcytoplasmatic (N/C) ratio, a micropapillary pattern, and increased nuclear atypia compared with classical PTC (Fig. 1) $[8,10]$. HVPTC is more common in women, and when compared with classical PTC tends to present at a higher stage with increased rates of regional and distant metastases as well as a higher mortality rate [7, 9, 11-18].

A systematic review of the existing literature describing HVPTC has yet to be conducted. Therefore, we performed a systematic review to unify the current literature in order to define the baseline clinicopathological characteristics of HVPTC and ascertain its prognostic implications.

\section{Materials and methods}

\section{Search strategy}

This systematic review queried four databases (PubMed, Scopus, Cochrane Library, and OVID) for any relevant studies for inclusion. This search included studies published from inception of the database to March 20th, 2020. This systematic review was conducted according to the Preferred Reporting Items for Systematic Reviews and Meta-Analyses (PRISMA) guidelines [19]. Search criteria included using subject headings or keywords: hobnail, thyroid, cancer, papillary, carcinoma, and micropapillary. References were then uploaded to EndNote (Clarivate Analytics, Philadelphia, PA, USA) and screened for relevance.

\section{Study selection and eligibility criteria}

Studies examining the prognostic significance of the HVPTC were included. Specific inclusion criteria included (a) examination of HVPTC with defined diagnostic criteria; (b) patient demographic data including age and sex; and (c) pathological tumor data. We elected to examine all HVPTC cases with any proportion of tumor with hobnail features, as Asioli et al. [9] demonstrated even those HVPTCs with $<30 \%$ hobnail features demonstrate a worse pattern of disease. Exclusion criteria include: (a) non-human studies; (b) reviews; (c) non-English language; (d) incomplete or nonextractable data; and (e) data represented in another study. Two reviewers (LBD and FY) independently assessed titles and abstracts of all studies to screen for articles satisfying the inclusion criteria. Articles not satisfying inclusion criteria were then excluded. Full-text assessments were then performed to ensure satisfaction with inclusion criteria, and any discrepancies were then reviewed by a third author (SAN) for determination.

\section{Critical appraisal and quality assessment}

Articles were critically appraised to assess the level of evidence using the Oxford Center for Evidence-Based Medicine criteria [20]. The risk of publication bias was assessed according to the Cochrane Handbook for

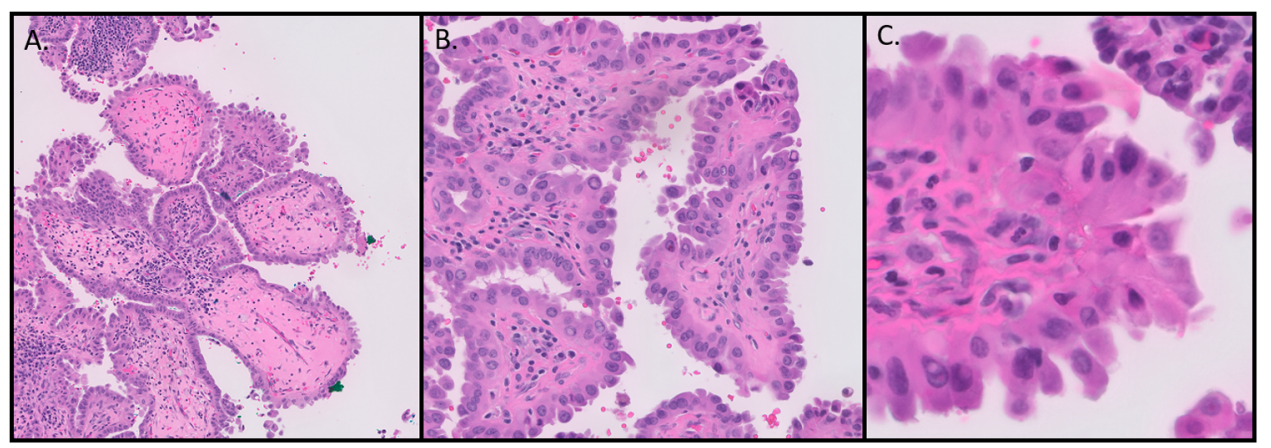

Fig. 1 The hobnail variant of papillary thyroid carcinoma exhibits (a) complex papillary and micropapillary structures (b) lined by neoplastic follicular cells with eosinophilic cytoplasm, loss of cellular polarity, apocrine snouting, and apically located nuclei, giving the cells the hallmark "hobnail" appearance. (c) Classic papillary thyroid carcinoma nuclear features are present, including intranuclear inclusions and nuclear grooves 
Systematic Reviews of Interventions 5.1.0 [21]. Two authors (LBD and FY) performed a pilot assessment on three studies to check for consistency of assessment. Both then performed independent risk assessment on the remaining studies. All disagreements were resolved by the way of discussion with a third author (SAN). Risk of bias items included the following: bias due to confounding, selection of participants into the study, classification of interventions, deviations from intended interventions, missing data, measurement of outcomes, and selection of the reported result. The risk of bias for each aspect is graded as "low," "unclear," or "high." (Supplemental Fig. 1).

\section{Data extraction}

Data extracted from studies included: author, publication year, study design, study characteristics, and patient demographics. Collected patient data included: age, sex, and comorbidities. Collected pathologic data included: stage, TNM classification, tumor size, presence of lymphovascular invasion (LVI), extrathyroidal extension (ETE) and/or necrosis, tumor percentage of hobnail features, distant metastasis, lymph node metastasis, identification of immunohistochemical and/or molecular markers, and follow-up data such as disease status, locoregional recurrence (LRR), and mortality. If only TNM classification was reported, but not overall tumor stage was given, these cases were then staged according to the 8th Edition of the American Joint Committee on Cancer (AJCC) Staging Manual [22].

\section{Statistical analysis}

All statistical analyses were performed using MedCalc 18.10.2 (MedCalc Software, bvba, Belgium). All analyses were weighted according to the number of patients affected. Meta-analysis of proportions was performed to pool the prevalence rate of HVPTC in all PTC cases. Heterogeneity was assessed by the $I^{2}$ test with the $I^{2}$ value directly correlating with degree of heterogeneity (lower $I^{2}$ value meaning lower degree of heterogeneity). A fixed statistical effect model was used if the $I^{2}$ value was $<50 \%$; for all other values, a random statistical effect model was used. The random effects model provides a more conservative estimate (i.e., with a wider confidence interval $[\mathrm{CI}]$ ), however the two models are often in agreement when there is no heterogeneity.

Finally, the Sterne and Egger tests were performed for further assessment of risk of publication bias [23, 24]. Potential publication bias was evaluated by visual inspection of the funnel plot and Egger's regression test. In a funnel plot, treatment effect is plotted on the horizontal axis and the standard error is on the vertical axis [25]. The vertical line represents the summary estimated derived using fixed-effect meta-analysis. Two diagonal lines represent (pseudo) $95 \%$ confidence limits (effect $\pm 1.96 \mathrm{SE}$ ) around the summary effect for each standard error on the vertical axis. These show the expected distribution of studies in the absence of heterogeneity or of selection bias. In the absence of heterogeneity, $95 \%$ of the studies should lie within the funnel defined by these diagonal lines. Publication bias results in asymmetry of the funnel plot.

We further provided a pooled summary of the studies meeting inclusion criteria. Of note, multiple studies provided patient-level data for which we were able to delineate patients $<30 \%$ and $\geq 30 \%$ hobnail features and patients $<55$ years old versus $\geq 55$ years old. We chose $30 \%$ hobnail features as a cutoff as Asioli et al. [8] first used this threshold as a diagnostic criterion for HVPTC, however later defined tumors with $<30 \%$ features to also have a poor prognosis. We used 55 years of age at diagnosis as a cutoff for staging, as per AJCC8, patients $\geq 55$ years of age are automatically classified as stage III or IV [22]. A Chi square test was used to compare features of two groups (HVPTC with $<30 \%$ versus $\geq 30 \%$ hobnail features; HVPTC $<55$ years old versus $\geq 55$ years old). If a significant difference was found for a particular variable, then an odds ratio (OR) with a 95\% CI was calculated by using a two-by-two frequency table where $a=$ no. of exposed cases, $b=$ no. of exposed non-cases, $c=$ no. of unexposed cases, $d=$ no. of unexposed non-cases, and $\mathrm{OR}=a d / b c$. A $p$ value of $<0.05$ was considered to indicate a statistically significant difference for all statistical tests.

\section{Results}

\section{Search results}

A total of 125 articles were identified through our literature search. Of these, 65 articles remained after removing duplicates. Initial screening by title and abstract left 27 articles for full-text review. A total of 16 studies met inclusion criteria and underwent quantitative analysis (Fig. 2) [7, 9, 11, 12, 17, 18, 26-35]. Based on the Oxford Level of Evidence, 13 of the 16 studies are deemed as level 4 evidence with the remaining 3 deemed as level 3 evidence. A funnel plot was generated to assess risk of publication bias and did not reveal any points outside the plot, indicating low publication bias (Supplemental Fig. 2).

\section{Overview of included studies}

This systematic review included 16 studies with a total of 124 patients with HVPTC (Table 1). Of the included patients, $81(65 \%)$ were female and $43(35 \%)$ male. The mean age at diagnosis was 52.3 years, with a range of 21-92 years. Mean follow-up time was 49.9 months, with 


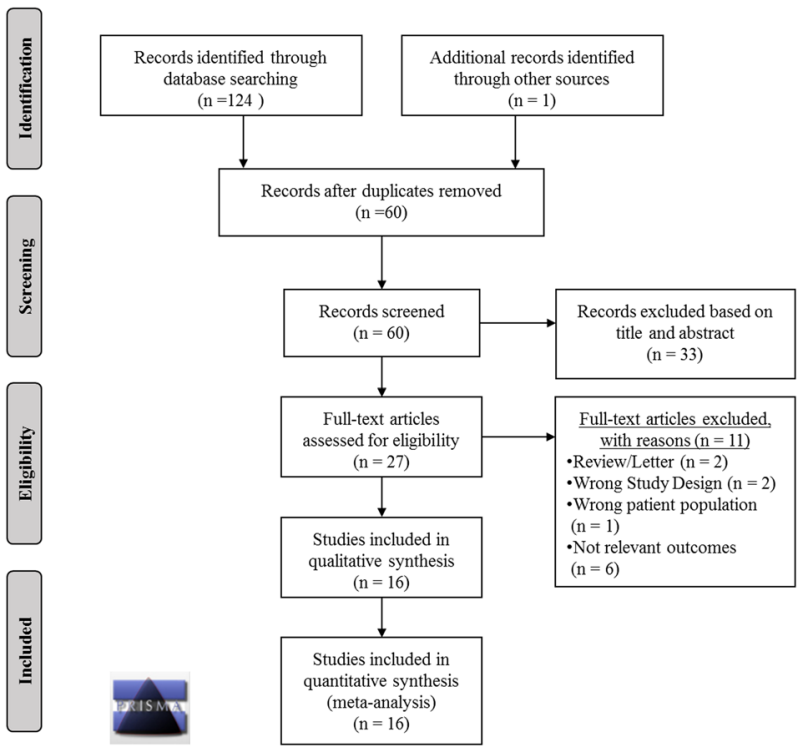

Fig. 2 Search strategy according to PRISMA guidelines

a range of 4-274 months. Of the eight studies that included prevalence data, the pooled overall prevalence of HVPTC in PTC was $1.08 \%$ (95\% 0.69-1.55) (Fig. 3) [9, 11, 12, 17, 18, 27, 31, 34]. Eighty-five patients had $\geq 30 \%$ hobnail component, 29 patients had $<30 \%$ hobnail component, and 25 cases did not have the percentage of hobnail features available for review. Of 114 cases that had tumor size data available, mean HVPTC tumor size was $3.1 \mathrm{~cm}$ (range $0.5-9 \mathrm{~cm}$ ). Twelve studies [12, 17, 18, 26-34] included focality of HVPTC, with 30 patients $(36 \%)$ having multifocal disease and 53 patients $(64 \%)$ having unifocal disease.

\section{Treatment}

All patients underwent thyroidectomy, with 95 (77\%) receiving a total thyroidectomy, $2(2 \%)$ receiving a hemithyroidectomy, and $26(21 \%)$ for which extent of thyroidectomy is unknown. In addition, 77 (63\%) patients received lymph node dissection; however, laterality and extent were not well-elucidated. Fifty-six (46\%) patients were reported to have received adjuvant RAI and $8(7 \%)$ received radiation therapy.

\section{Clinicopathologic features and outcomes}

The prevalence of three high-risk pathologic features (LVI, ETE, and necrosis) were examined (Table 2). Of the 13 studies reporting LVI, 69/111 (62\%) tumors presented with LVI [7, 9, 11, 17, 18, 26, 27, 29-34]. Of the 11 studies reporting ETE, 32/64 (50\%) tumors presented with ETE [7, 11, 12, 26, 27, 29, 31-35]. Of 104 cases with T-category available, 9 were classified as $\mathrm{T} 4 \mathrm{a}$ or $\mathrm{T} 4 \mathrm{~b}$, indicating extensive ETE to larynx, trachea, recurrent laryngeal nerve (T4a), or invading prevertebral fascia or encasing carotid arteries (T4b). Of the 8 studies reporting necrosis, 9/58 (16\%) tumors presented with necrosis $[7,9,12,17,27,30-32]$. B-type raf kinase $(B R A F)$ p.V600E mutations were present in 62/85 (73\%) cases with evaluable mutational data. Forty-six (47\%) cases were stage I, 13 (14\%) were stage II, 18 (19\%) were stage III, and $20(21 \%)$ were stage IV at presentation.

Multiple outcome measures, such as rates of LRR, persistent disease, lymph node metastasis, distant metastasis, and overall survival were also evaluated. Of the 13 studies reporting LRR or persistent disease, 43/121 (36\%) patients developed LRR or had persistent disease. The overall rate of lymph node metastasis was $80 / 122(66 \%)$. The overall rate of distant metastasis was 27/116 (23\%). Sites of distant metastasis included lung (18/27 [67\%]), bone (10/27 [37\%]), liver (5/27 [19\%]), brain (4/27 [15\%]), soft tissue/ muscle $(5 / 27$ [19\%]), pancreas (1/27 [4\%]), spinal cord $(1 / 27$ [4\%]), epiglottis/larynx (2/27 [8\%]), and nasopharynx (2/27 [8\%]). Of the 11 studies reporting survival status of their patients $[11,12,17,18,26,27,29-31,34,35], 53 / 96$ $(55 \%)$ had no evidence of disease, 24/96 (25\%) were alive with disease, 10/95 (10\%) died of disease, and 9/96 (9\%) were lost to follow-up.

\section{Comparison of $<30 \%$ and $\geq 30 \%$ hobnail}

Tumors with $\geq 30 \%$ hobnail morphology had an odds ratio of 2.6 (95\% CI 1.1-6.2) of developing lymph node metastasis over tumors with $<30 \%$ hobnail morphology. No significant differences were seen in rates of distant metastasis or in highrisk pathological features such as LVI, ETE, and necrosis. $B R A F$ mutation rates were similar in both groups (Table 3 ).

\section{Comparison of HVPTC $<55$ and $\geq 55$ years old}

Patients $<55$ and $\geq 55$ years of age with HVPTC were compared. Patients $\geq 55$ years old had an increased likelihood of developing distant metastasis (OR 4.5, 95\% CI 1.6-12.6) and having LVI (OR 4.7, 95\% CI 1.8-12.7) as a high-risk pathological feature compared with patients $<55$ years old. No significant differences were seen in rates of lymph node metastasis or BRAF mutation rates. Other high-risk pathological features such as ETE and necrosis were not significantly different (Table 4).

\section{Discussion}

In part owing to the recent identification of HVPTC, tumor characteristics and clinical implications of this high-risk PTC variant remain poorly understood. The aim of our 


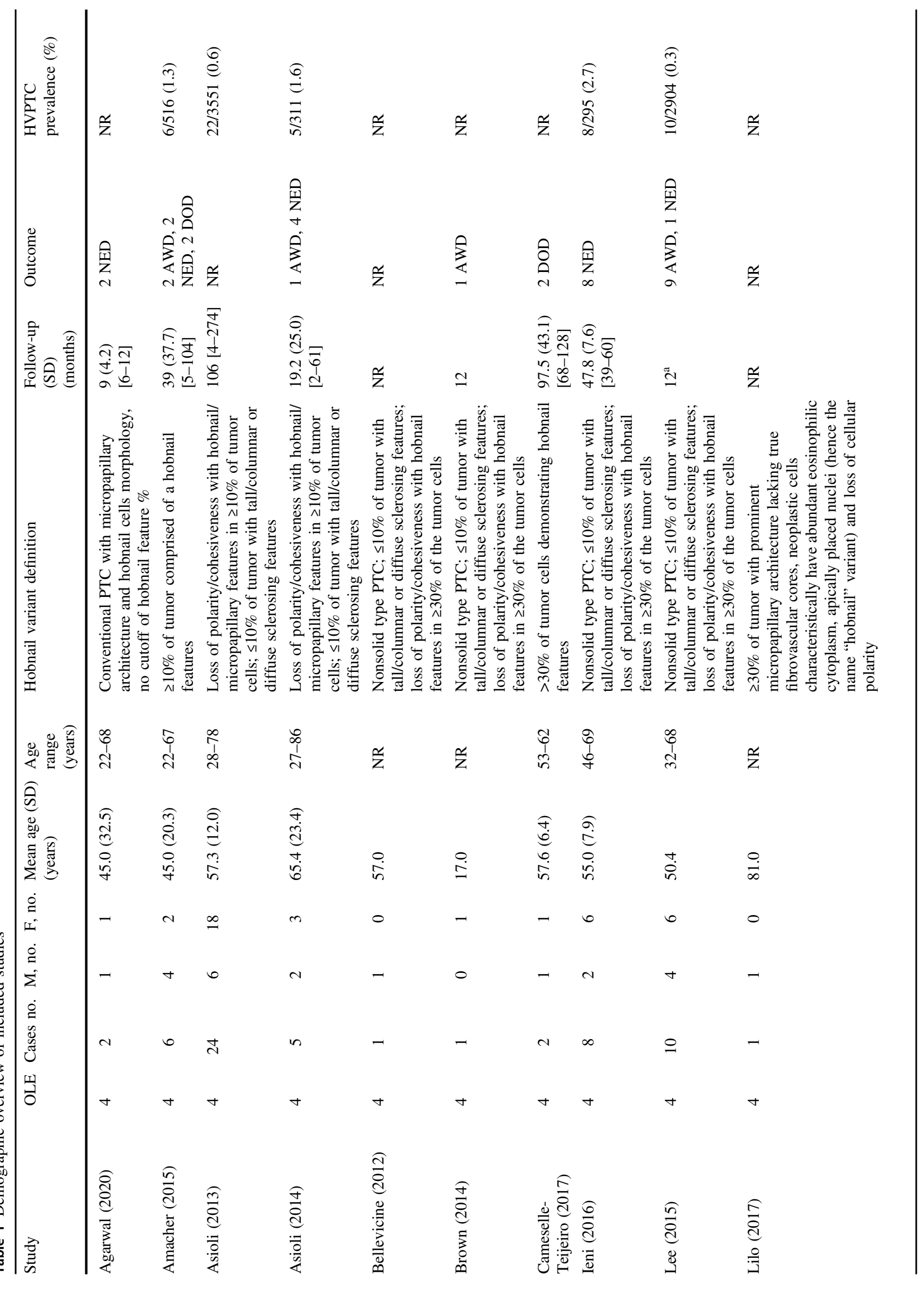




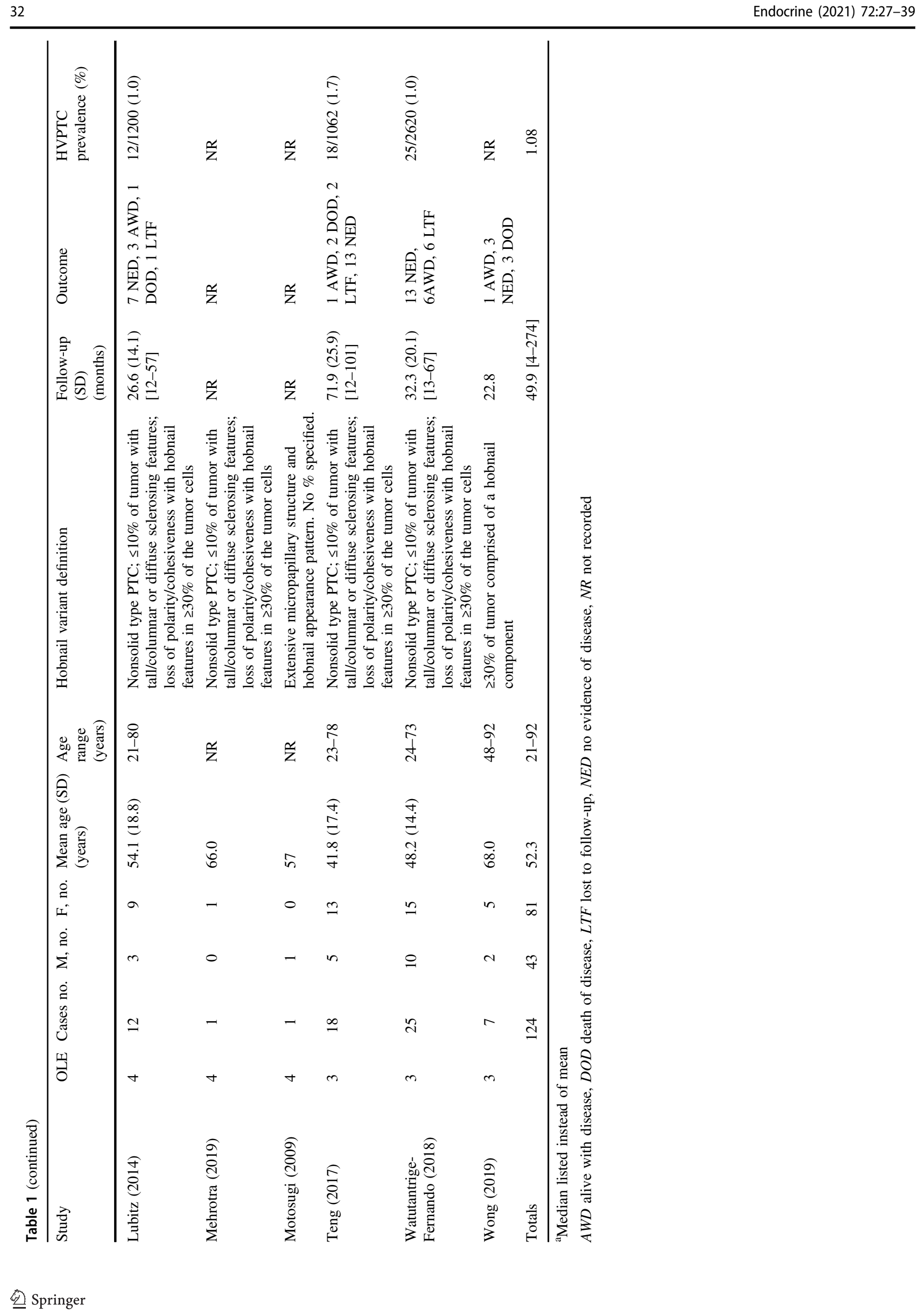




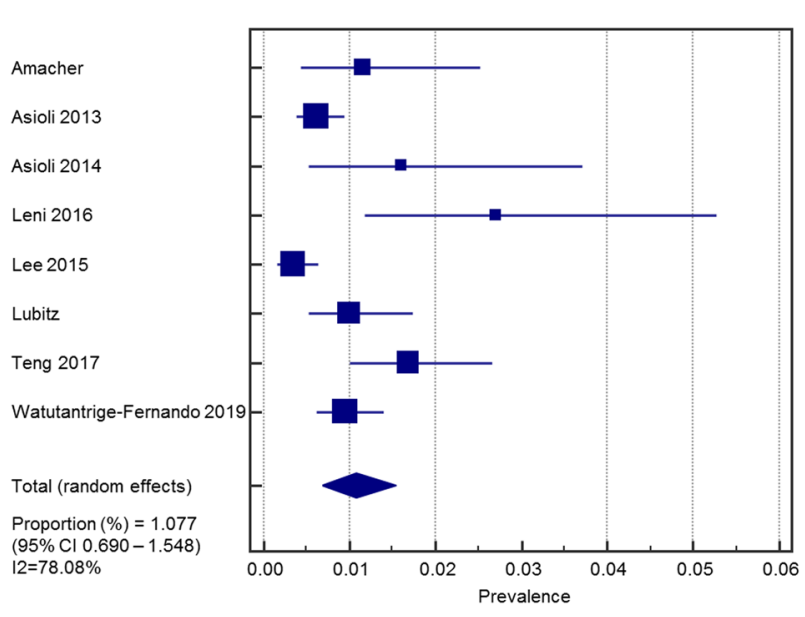

Fig. 3 Forest plot demonstrating pooled prevalence of HVPTC in PTC cases

study was to systematically review the current literature to analyze clinicopathological features of HVPTC in order to further our understanding of this disease. Our study demonstrated an overall prevalence of $1.08 \%$ of HVPTC in PTC cases. HVPTC remains a rare but aggressive variant of PTC as evidenced by the greater rate of high-risk pathological features (LVI, ETE, and necrosis) and worse outcomes than that reported of classical PTC.

We have compared our findings for HVPTC to that of the reported literature values regarding classical PTC (Table 5). PTC is diagnosed at a mean age of 45 and shows a female predominance of $60-80 \%$ [36]. We found that HVPTC is diagnosed in older individuals with a mean age at diagnosis of 52.3 years and with a slight female predominance $(65 \%$ female) similar to that of classical PTC. The mean size of all thyroid cancers at diagnosis has been reported to be $1.9 \mathrm{~cm}$ with $82 \%$ of thyroid cancers $3.0 \mathrm{~cm}$ or smaller in size [37]. Comparatively, our study demonstrated HVPTC tumors to have a larger mean size of $3.1 \mathrm{~cm}$. Increased tumor size in classical PTC has been found to predict poor prognosis $[38,39]$. Thus, the larger mean size of HVPTC at presentation highlights advanced T-category in comparison with classical PTC.

Well established high-risk pathologic characteristics of PTC include, but are not limited to, LVI, ETE, and necrosis. In a single institution retrospective review, Hay et al. [40] found a total of 328/2444 (13\%) PTC patients to have the primary tumor invade into surrounding extrathyroidal soft tissue. Comparatively, our study demonstrated HVPTC tumors to have a much greater proportion of ETE at 50\%, indicative of the more aggressive nature of HVPTC. Furthermore, a large retrospective cohort series by Xing et al. [41] examining classical PTC patients found a significant association between $B R A F$ mutations and aggressive features and mortality, with $845 / 1849$ (45.7\%) patients possessing a positive $B R A F$ mutation. Our study demonstrated a substantially greater percentage of tumors possessing BRAF mutation (73\%). This demonstrates the greater risk of cancer-related mortality in HVPTC patients as BRAF mutational genotypes are associated with higher rates of disease recurrence, decreased disease-free survival, and decreased overall survival $[42,43]$. Targeted therapies for BRAF-mutated PTC, such as BRAF-specific inhibitors like vemurafenib and dabrafenib, have had promising results $[44,45]$. In BRAF-mutated anaplastic thyroid carcinoma, these inhibitors, in combination with mitogen-activated protein kinase kinase inhibitors, have led to an overall response rate of $69 \%$ [46]. Consequently, the dual blockage of the MAPK pathway may prove to be significantly efficacious in HVPTC and is an area of ongoing investigation [46]. Additionally, the role of BRAF inhibition to sensitize RAI-refractory thyroid cancers to RAI may also play a role in the management of advanced HVPTC, with promising evidence revealing that vemurafenib restored RAI uptake in RAI-refractory BRAF V600E mutated thyroid cancers [47]. Other molecular markers such as RET/PTC, TERT, p53, and IPK3CA were not consistently evaluated in the majority of studies, however can be the focus for future molecular characterization of HVPTC in order to improve prognostic risk stratification and allow for development of potential targeted therapeutic options.

In regards to disease outcomes, HVPTC portends worse outcomes as compared with classical PTC. Differentiated thyroid carcinomas, including classical PTC, will develop local or regional metastasis in $5-20 \%$ of cases, and distant metastases in $10-15 \%$ of patients, notably to the lungs and bones $[48,49]$. We found that HVPTC tends to have an increased local and regional recurrence rate, with 43/121 (36\%) patients found to have recurrence in the 13 studies analyzed. In addition, the overall rate of distant metastasis was $27 / 116(23 \%)$, with lung and bone being most commonly affected.

The impact of percentage of hobnail features has previously been evaluated. Asioli et al. [9] reviewed 24 HVPTC cases and stratified them into two groups: (1) PTC cases with $\geq 30 \%$ hobnail/micropapillary features and (2) PTC cases with $<10 \%$ hobnail/micropapillary features. Their analysis found increased hobnail/micropapillary features were associated with an increased risk of death, distant metastases, and decreased disease-free survival. No significant differences were found in regards to gender, age, tumor size, LVI, ETE, RAI therapy and stage between the groups. Similarly, Watutantrige-Fernando et al. [18] analyzed differences between two groups: PTC cases with $\geq 30 \%$ hobnail component (16/25) and PTC cases with $<30 \%$ hobnail component (9/25). Interestingly, their study found no statistically significant differences between the groups with regards to lymph node involvement, distant metastasis, and outcomes. They also reported no differences 


\begin{tabular}{|c|c|c|c|c|c|c|c|c|c|c|c|c|}
\hline 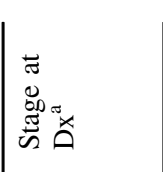 & $\mid \begin{array}{l}= \\
\vec{n} \\
\vdots \\
=-5\end{array}$ & 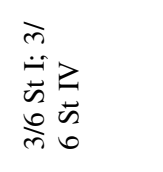 & 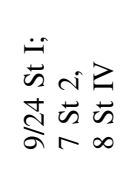 & $\frac{a}{z}$ & 弚 & $\frac{a}{z}$ & $\begin{array}{l}\text { B } \\
\overline{0} \\
\text { I } \\
I\end{array}$ & 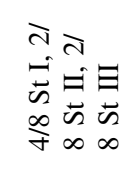 & 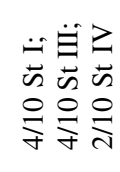 & 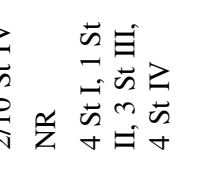 & 夽 兰 & 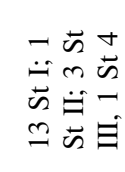 \\
\hline$\sum$ & $\delta$ & $\stackrel{\text { m }}{\circ}$ & 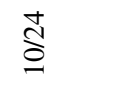 & 弚 & 苂 & $\Xi$ & ป & $\stackrel{\infty}{\circ}$ & $\stackrel{0}{\partial}$ & $\tilde{z} \frac{N}{m}$ & 兑 & $\stackrel{\lambda}{\lambda}^{\infty}$ \\
\hline$\sum_{z}^{\pi}$ & I & $\stackrel{\circ}{\circ}=$ & 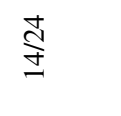 & $\stackrel{n}{m}$ & 艺 & $\Xi$ & $\frac{4}{2}$ & $\stackrel{\infty}{\lambda}$ & $\frac{\wp}{\infty}$ & 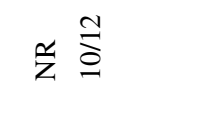 & $\Xi \Xi$ & 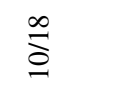 \\
\hline 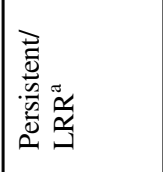 & I & 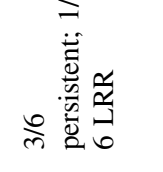 & 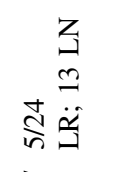 & $\begin{array}{l}\stackrel{a}{a} \\
\stackrel{a}{=} \\
=\end{array}$ & 弚 & $\Xi$ & $\underset{\sim}{Z}$ & $\stackrel{\infty}{\partial}$ & $\begin{array}{l}\text { Z } \\
\stackrel{0}{\Xi}\end{array}$ & 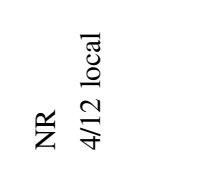 & 艺 & $\stackrel{\infty}{=}$ \\
\hline 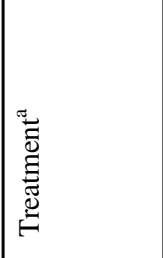 & $\begin{array}{l}E \\
\text { I }\end{array}$ & 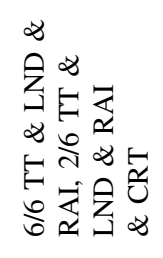 & 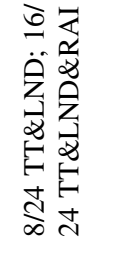 & 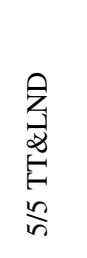 & 苂 & 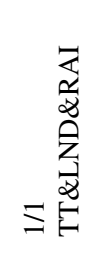 & 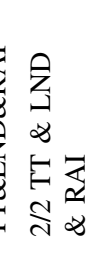 & 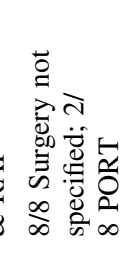 & 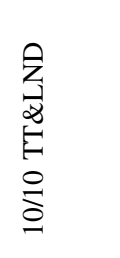 & 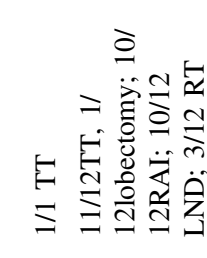 & 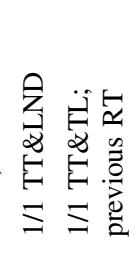 & 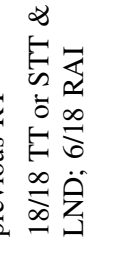 \\
\hline 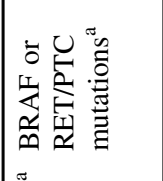 & 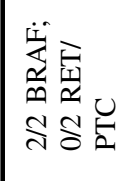 & 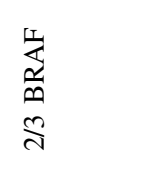 & $\frac{n}{z}$ & $\begin{array}{l}\frac{1}{2} \\
\frac{n}{m} \\
m \\
m\end{array}$ & 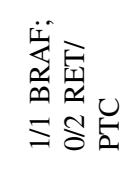 & & 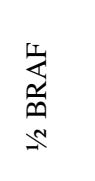 & 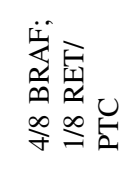 & $\begin{array}{l}\frac{1}{\grave{2}} \\
\frac{\pi}{\infty} \\
\stackrel{\infty}{\infty}\end{array}$ & 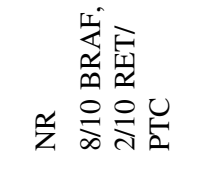 & $\tilde{z} \tilde{z}$ & 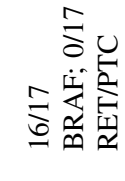 \\
\hline 离 & 亗 & $\stackrel{\circ}{m}$ & $\stackrel{\check{a}}{\partial}$ & $\frac{n}{m}$ & 芫 & 学 & $\approx$ & $\stackrel{\infty}{\partial}$ & $\stackrel{\circ}{\stackrel{i}{\lambda}}$ & ذ气 & 芫 & 妾 \\
\hline 离 & $\frac{8}{2}$ & $\stackrel{\circ}{f}$ & $\frac{a}{z}$ & $\stackrel{n}{8}$ & 弚 & $\Xi$ & $\frac{a}{z}$ & 弚 & $\stackrel{ }{i}$ & $\Xi \stackrel{N}{N}$ & $\Xi \Xi$ & $\bigsqcup_{0}^{\infty}$ \\
\hline$\frac{\tilde{\sigma}}{>}$ & $\delta$ & $\frac{\tilde{z}}{z}$ & $\stackrel{n}{\stackrel{\Xi}{\Xi}}$ & $\stackrel{n}{f}$ & 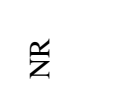 & $\Xi$ & $\frac{4}{4}$ & $\stackrel{\infty}{\forall}$ & $\stackrel{\circ}{\infty}$ & $\Xi \frac{\tilde{\nu}}{n}$ & $\Xi \Xi$ & $\stackrel{\infty}{\lambda}$ \\
\hline 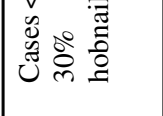 & 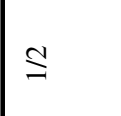 & $\stackrel{\circ}{\Im}$ & $\stackrel{+}{\Xi}$ & $\frac{n}{m}$ & 号 & $\bar{\delta}$ & ร & $\stackrel{\infty}{\partial}$ & $\stackrel{Ð}{\partial}$ & 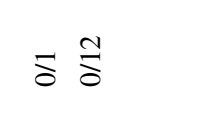 & 关芫 & $\stackrel{\infty}{\partial}$ \\
\hline 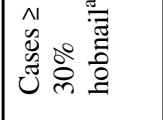 & $S$ & $\stackrel{2}{a}$ & 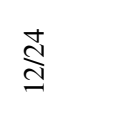 & $\stackrel{n}{a}$ & 苂 & $\Xi$ & ล & $\stackrel{\infty}{\infty}$ & $\stackrel{\circ}{9}$ & $\Xi \stackrel{\Xi}{\Xi}$ & 妾 芫 & $\stackrel{\infty}{\stackrel{\infty}{\infty}}$ \\
\hline 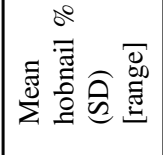 & 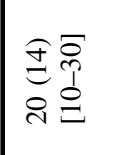 & 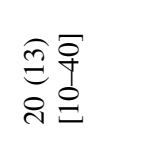 & 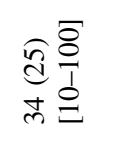 & 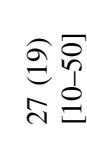 & & $\therefore$ & 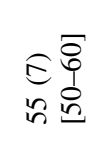 & 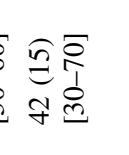 & $\tilde{z}$ & 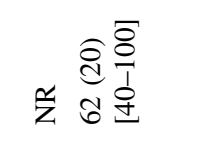 & 苂关 & 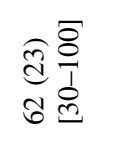 \\
\hline 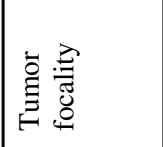 & 己 & 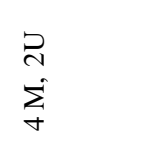 & 弚 & $\begin{array}{l}q \\
\Sigma \\
\Sigma\end{array}$ & $\supseteq$ & $?$ & $\stackrel{2}{2}$ & $\begin{array}{l}R \\
\Sigma\end{array}$ & $\stackrel{?}{\varrho}$ & $\supseteq \tilde{z}$ & 己 & $\begin{array}{l}D \\
n \\
\sum_{m}\end{array}$ \\
\hline 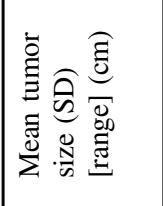 & 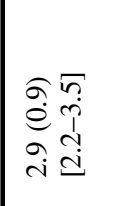 & 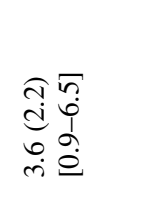 & $\begin{array}{l}\underset{I}{\stackrel{T}{E}} \\
\stackrel{i}{\Xi} \\
a\end{array}$ & 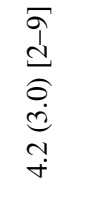 & $\stackrel{\circ}{i}$ & ले & 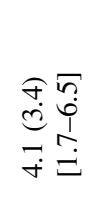 & 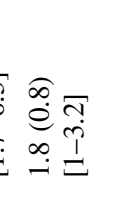 & $\begin{array}{l}\text { F } \\
\stackrel{0}{0} \\
\stackrel{0}{0} \\
\stackrel{m}{-}\end{array}$ & 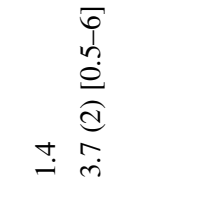 & min & $\underset{\substack{n \\
\stackrel{n}{\Xi}}}{\stackrel{\sqrt{n}}{n}}$ \\
\hline & 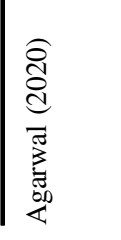 & 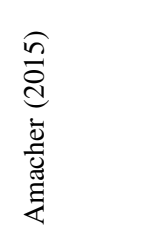 & $\begin{array}{l}\widehat{m} \\
\stackrel{0}{a} \\
: \frac{0}{0} \\
\frac{0}{2}\end{array}$ & $\begin{array}{l}\frac{9}{2} \\
\frac{d}{2} \\
: \frac{0}{0} \\
\frac{0}{4}\end{array}$ & 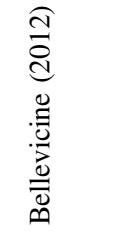 & 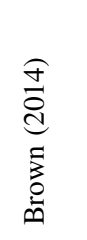 & 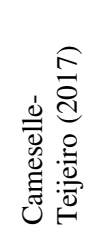 & 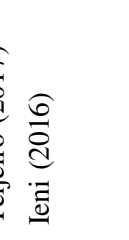 & 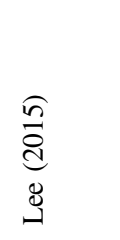 & 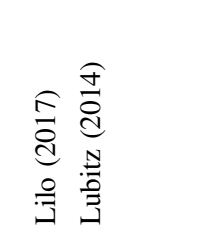 & 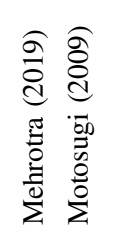 & 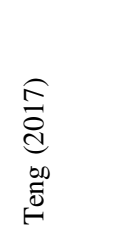 \\
\hline
\end{tabular}




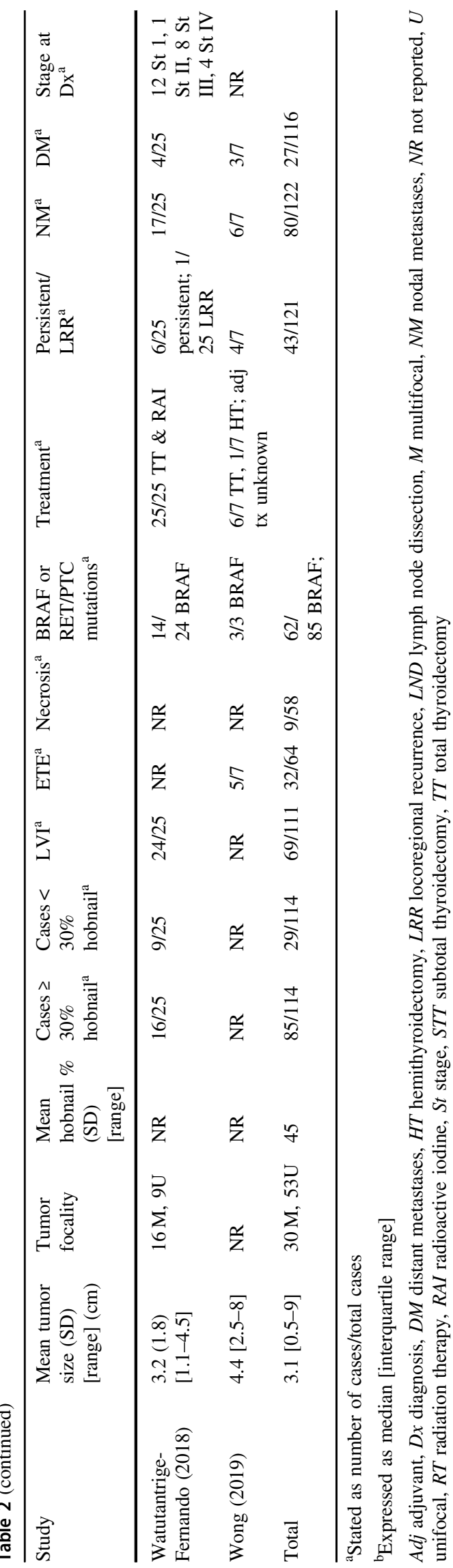

Table 3 Comparison of HVPTC with $<30 \%$ versus $\geq 30 \%$ hobnail features $^{\mathrm{a}}$

\begin{tabular}{|c|c|c|c|}
\hline & $\begin{array}{l}\text { HVPTC }<30 \% \\
\text { hobnail features } \\
(n=28)\end{array}$ & $\begin{array}{l}\text { HVPTC } \geq 30 \% \\
\text { hobnail features } \\
(n=85)\end{array}$ & $p$ value \\
\hline LN metastasis & & & 0.034 \\
\hline Yes & 14 & 59 & \\
\hline No & 14 & 23 & \\
\hline Distant metastasis & & & 0.323 \\
\hline Yes & 4 & 20 & \\
\hline No & 23 & 64 & \\
\hline BRAF mutation & & & 0.595 \\
\hline Yes & 11 & 31 & \\
\hline No & 4 & 16 & \\
\hline$L V I$ & & & 0.530 \\
\hline Yes & 9 & 27 & \\
\hline No & 7 & 30 & \\
\hline ETE & & & 0.985 \\
\hline Yes & 3 & 16 & \\
\hline No & 4 & 21 & \\
\hline Necrosis & & & 0.638 \\
\hline Yes & 3 & 6 & \\
\hline No & 15 & 43 & \\
\hline
\end{tabular}

${ }^{a}$ Incomplete data not included. Variables may not sum to total $\mathrm{n}$ because of different variability of presenting information

ETE extrathyroidal extension, HVPTC hobnail variant of papillary thyroid carcinoma, $L N$ lymph node, $L V I$ lymphovascular invasion

in age, tumor size, multifocality, staging, and mutational status. Our pooled analysis found an odds ratio of 2.6 for lymph node metastasis when comparing cases with $\geq 30 \%$ hobnail features versus cases with $<30 \%$ hobnail features, however no significant differences were found in rates of distant metastasis or the previously discussed high-risk pathological characteristics. This may indicate that proportion of hobnail component is prognostically significant and may influence risk of locoregional disease; however, PTC cases $<30 \%$ hobnail features should still be consistently considered an aggressive variant of PTC and further analysis is warranted.

Patients $\geq 55$ years old with HVPTC had significantly higher rates of LVI and distant metastasis than patients $<55$ years old ( $35 \%$ vs $11 \%, p=0.003)$, however there were no significant differences in lymph node metastasis rates, LVI, $B R A F$ mutation status or necrosis. The rates of distant metastasis in patients $<55$ years old $(11 \%)$ were comparable to that reported in the literature of differentiated thyroid carcinoma $(10-15 \%)[48,49]$. Both cohorts also had high rates of lymph node metastasis at 55\% in $<55$ years old group and $72 \%$ in the $\geq 55$ years old group, which is greater than reported median values of lymph node metastasis (20-50\%) in classical PTC [50]. In conclusion, although 
age may predict increased rates of distant metastasis, patients younger than 55 years old still had a comparable risk of lymph node metastasis, $B R A F$ mutation and necrosis to patients $\geq 55$ years old. Consequently, patients of any age with HVPTC require close attention.

Overall, these findings highlight the aggressive nature of HVPTC in comparison with classical PTC. These patients tend to have larger tumors, high-risk pathologic characteristics, and worse outcomes such as higher rates of LRR, distant metastasis, and death. Treatment specific to HVPTC warrants further investigation, as the role of RAI in this patient

Table 4 Comparison of HVPTC by age ${ }^{\mathrm{a}}$

\begin{tabular}{lccc}
\hline & $<55$ YO $(n=56)$ & $\geq 55$ YO $(n=48)$ & $p$ value \\
\hline LN metastasis & 31 & 34 & 0.094 \\
$\quad$ Yes & 24 & 13 & \\
$\quad$ No & & & \\
Distant metastasis & & 17 & \\
$\quad$ Yes & 6 & 31 & 0.003 \\
No & 49 & & \\
BRAF mutation & & 19 & 0.305 \\
Yes & 15 & 6 & \\
No & 9 & & \\
LVI & & 24 & 0.625 \\
Yes & 11 & 12 & \\
No & 26 & & \\
ETE & & 9 & \\
Yes & 10 & 10 & \\
No & 15 & 6 & \\
Necrosis & & 28 & \\
Yes & 1 & & \\
No & 23 & & \\
\hline
\end{tabular}

${ }^{a}$ Incomplete data not included. Variables may not sum to total $\mathrm{n}$ because of different variability of presenting information

ETE extrathyroidal extension, HVPTC hobnail variant of papillary thyroid carcinoma, $L N$ lymph node, $L V I$ lymphovascular invasion population is still unknown. Morandi et al. [16] reported HVPTC to have a poor response to radioiodine therapy, with only 6/18 patients demonstrating good iodine uptake and shrinkage of metastatic disease. As more knowledge about this variant of PTC is discovered, targeted therapies may develop to better treat the disease. Further prospective studies are needed to better assess pertinent diagnostic features and genetic drivers behind HVPTC in order to best risk stratify and maximize therapeutic intervention.

\section{Limitations}

Limitations of this study include paucity of data directly comparing HVPTC with classical PTC, heterogeneity in definitions of hobnail variant, and different follow-up times that can skew reported outcomes. Additionally, our study aimed to exam molecular markers of HVPTC and could only successfully evaluate BRAF mutational status in 8 studies. Other molecular markers that were considered included RET/PTC, TERT, p53, and IPK3CA, but these were not consistently reported by a majority of studies and may represent a possible focus of future studies. Also, our systematic review included studies of low-level evidence (i.e. case reports) given the rarity of HVPTC. Additionally, certain studies with novel data were excluded due to an overlap in patients previously reported in other studies which reduces overall data volume.

\section{Conclusions}

In this systematic review, we found that the HVPTC is associated with more aggressive clinicopathological features and a poorer prognosis as compared with classical PTC. This is seen by increased mean tumor size at diagnosis, higher rates of LVI, ETE, tumor necrosis, and $B R A F$ mutation status. In addition, poorer outcomes as demonstrated by higher rates of LRR, lymph node metastasis, and distant metastasis compared to reports in the literature for
Table 5 Comparison of PTC and HVPTC as reported in the literature

\begin{tabular}{lll}
\hline & PTC & HVPTC \\
\hline Mean age at diagnosis & 45 years [26] & 52.3 years \\
Gender predominance & Female, 60-80\% [26] & Female, 65\% \\
Mean tumor size & $2.1 \mathrm{~cm} \mathrm{[28]}$ & $3.1 \mathrm{~cm}$ \\
Rate of ETE & $13 \%[28]$ & $50 \%$ \\
Rate of BRAF mutation & $45.7 \%[31]$ & $73 \%$ \\
Rate of recurrence & $8 \%[28]$ (mean 60 months) & $36 \%$ (mean 48.9 months) \\
Rate of distant metastasis & $12.4 \%$ (range 0-168 months) [33] & $23 \%$ (range 4-274 months) \\
Disease mortality & $4 \%[28]$ & $10 \%$ \\
\hline
\end{tabular}

ETE extrathyroidal extension, HVPTC Hobnail variant papillary thyroid carcinoma, PTC papillary thyroid carcinoma 
classical PTC may support the aggressive nature of this hobnail variant. As HVPTC cases with $<30$ and $\geq 30 \%$ hobnail component did not significantly differ in rates of distant metastasis or presence of high-risk features, further refinement of diagnostic criteria regarding percentage of hobnail tumor involvement is warranted. Future investigation is required to better characterize these clinicopathological features and the resulting impact on response to treatment.

Author contributions Concept and design: Donaldson, Yan, Nguyen, and Day. Acquisition, analysis, or interpretation of data: Donaldson and Yan. Drafting of Manuscript: Donaldson, Yan, Morgan, and Morgan. Critical revision of manuscript: Morgan, Nguyen, Fernandes, Kaczmar, Jester, and Day. Statistical analysis: Yan and Nguyen. Supervision: Nguyen, Fernandes, Kaczmar, Jester, and Day.

\section{Compliance with ethical standards}

Conflict of interest The authors declare that they have no conflict of interest.

Publisher's note Springer Nature remains neutral with regard to jurisdictional claims in published maps and institutional affiliations.

\section{References}

1. J. Ferlay, M. Colombet, I. Soerjomataram, C. Mathers, D.M. Parkin, M. Piñeros, A. Znaor, F. Bray, Estimating the global cancer incidence and mortality in 2018: GLOBOCAN sources and methods. Int. J. Cancer. 144(8), 1941-1953 (2019). https://doi. org/10.1002/ijc.31937

2. S.A. Hundahl, I.D. Fleming, A.M. Fremgen, H.R. Menck,, A National Cancer Data Base report on 53,856 cases of thyroid carcinoma treated in the U.S., 1985-1995 [see commetns]. Cancer 83(12), 2638-2648 (1998). https://doi.org/10.1002/(sici)10970142(19981215)83:123.0.co;2-1

3. National Cancer Institute: Cancer Stat Facts: Thyroid Cancer. https://seer.cancer.gov/statfacts/html/thyro.html (2020). Accessed June 82020

4. R.L.R. Osamura, G. Kloppel, J. Rosai: WHO Classification of Tumours of Endocrine Organs. WHO Classification of Tumours, 4th edn, vol. 10 (2017)

5. M. Pusztaszeri, M. Auger, Update on the cytologic features of papillary thyroid carcinoma variants. Diagn. Cytopathol. 45(8), 714-730 (2017). https://doi.org/10.1002/dc.23703

6. K. Kakudo, W. Tang, Y. Ito, I. Mori, Y. Nakamura, A. Miyauchi, Papillary carcinoma of the thyroid in Japan: subclassification of common type and identification of low risk group. J. Clin. Pathol. 57 (10), 1041-1046 (2004). https://doi.org/10.1136/jcp.2004.017889

7. U. Motosugi, S. Murata, K. Nagata, M. Yasuda, M. Shimizu, Thyroid papillary carcinoma with micropapillary and hobnail growth pattern: a histological variant with intermediate malignancy? Thyroid 19(5), 535-537 (2009). https://doi.org/10.1089/ thy. 2008.0271

8. S. Asioli, L.A. Erickson, T.J. Sebo, J. Zhang, L. Jin, G.B. Thompson, R.V. Lloyd, Papillary thyroid carcinoma with prominent hobnail features: a new aggressive variant of moderately differentiated papillary carcinoma. A clinicopathologic, immunohistochemical, and molecular study of eight cases. Am. J. Surg. Pathol. 34(1), 44-52 (2010). https://doi.org/10.1097/PAS.0b013e3181c46677
9. S. Asioli, L.A. Erickson, A. Righi, R.V. Lloyd, Papillary thyroid carcinoma with hobnail features: histopathologic criteria to predict aggressive behavior. Hum. Pathol. 44(3), 320-328 (2013). https:// doi.org/10.1016/j.humpath.2012.06.003

10. K.S. Wong, T.Y. Chen, S.E. Higgins, B.E. Howitt, J.H. Lorch, E. K. Alexander, E. Marqusee, N.L. Cho, M.A. Nehs, G.M. Doherty, J.A. Barletta, A potential diagnostic pitfall for hobnail variant of papillary thyroid carcinoma. Histopathology 76(5), 707-713 (2020). https://doi.org/10.1111/his. 14042

11. C.C. Lubitz, K.P. Economopoulos, A.C. Pawlak, K. Lynch, D. Dias-Santagata, W.C. Faquin, P.M. Sadow, Hobnail variant of papillary thyroid carcinoma: an institutional case series and molecular profile. Thyroid 24(6), 958-965 (2014). https://doi.org/ 10.1089/thy.2013.0573

12. A.M. Amacher, B. Goyal, J.S. Lewis Jr, S.K. El-Mofty, R.D. Chernock, Prevalence of a hobnail pattern in papillary, poorly differentiated, and anaplastic thyroid carcinoma: a possible manifestation of high-grade transformation. Am. J. Surg. Pathol. 39(2), 260-265 (2015). https://doi.org/10.1097/pas.0000000000000329

13. L.S. Lino-Silva, H.R. Domínguez-Malagón, C.H. Caro-Sánchez, R.A. Salcedo-Hernández, Thyroid gland papillary carcinomas with "micropapillary pattern," a recently recognized poor prognostic finding: clinicopathologic and survival analysis of 7 cases. Hum. Pathol. 43(10), 1596-1600 (2012). https://doi.org/10.1016/ j.humpath.2011.10.027

14. S.Y. Chung, J.S. Lee, H. Lee, S.H. Park, S.J. Kim, H.S. Ryu, Cytomorphological factors and BRAF mutation predicting risk of lymph node metastasis in preoperative liquid-based fine needle aspirations of papillary thyroid carcinoma. Acta Cytol. 57(3), 252-258 (2013). https://doi.org/10.1159/000343617

15. Y. Bai, K. Kakudo, Y. Li, Z. Liu, T. Ozaki, Y. Ito, M. Kihara, A. Miyauchi, Subclassification of non-solid-type papillary thyroid carcinoma identification of high-risk group in common type. Cancer Sci. 99(10), 1908-1915 (2008). https://doi.org/10.1111/j. 1349-7006.2008.00908.x

16. L. Morandi, A. Righi, F. Maletta, P. Rucci, F. Pagni, M. Gallo, S. Rossi, L. Caporali, A. Sapino, R.V. Lloyd, S. Asioli, Somatic mutation profiling of hobnail variant of papillary thyroid carcinoma. Endocr.-Relat. Cancer 24(2), 107-117 (2017). https://doi. org/10.1530/erc-16-0546

17. A. Ieni, V. Barresi, R. Cardia, L. Licata, F. Di Bari, S. Benvenga, G. Tuccari, The micropapillary/hobnail variant of papillary thyroid carcinoma: a review of series described in the literature compared to a series from one southern Italy pathology institution. Rev. Endocr. Metab. Disord. 17(4), 521-527 (2016). https://doi. org/10.1007/s11154-016-9398-4

18. S. Watutantrige-Fernando, F. Vianello, S. Barollo, L. Bertazza, F. Galuppini, E. Cavedon, S. Censi, C. Benna, E.C. Ide, A. Parisi, D. Nacamulli, M. Iacobone, G. Pennelli, C. Mian, The hobnail variant of papillary thyroid carcinoma: clinical/molecular characteristics of a large monocentric series and comparison with conventional histotypes. Thyroid 28(1), 96-103 (2018). https:// doi.org/10.1089/thy.2017.0248

19. D. Moher, A. Liberati, J. Tetzlaff, D.G. Altman; The, P.G., Preferred reporting items for systematic reviews and meta-analyses: the PRISMA statement. PLOS Med. 6(7), e1000097 (2009). https://doi.org/10.1371/journal.pmed.1000097

20. OCEBM Levels of Evidence Working Group*: The Oxford Levels of Evidence 2. https://www.cebm.net/index.aspx $?=05653$. Accessed Apr 232020

21. J.A. Sterne, M.A. Hernán, A. McAleenan, B.C. Reeves and J. P. Higgins: Assessing risk of bias in a non-randomized study. In: Cochrane Handbook for Systematic Reviews of Interventions. 2nd edn, pp. 621-641 (2019). https://doi.org/10.1002/ 9781119536604.ch25 
22. E.S. Amin MB, F. Greene, D.R. Byrd, R.K. Brookland, M.K. Washington, J.E. Gershenwald, C.C. Compton, K.R. Hess, et al.: AJCC Cancer Staging Manual (8th edition). Springer International Publishing: American Joint Commission on Cancer, (2017)

23. M. Egger, G.D. Smith, M. Schneider, C. Minder, Bias in metaanalysis detected by a simple, graphical test. BMJ 315(7109), 629-634 (1997). https://doi.org/10.1136/bmj.315.7109.629

24. J.A. Sterne, M. Egger, Funnel plots for detecting bias in meta-analysis: guidelines on choice of axis. J. Clin. Epidemiol. 54(10), 1046-1055 (2001). https://doi.org/10.1016/s0895-4356(01)00377-8

25. J.A.C. Sterne, A.J. Sutton, J.P.A. Ioannidis, N. Terrin, D.R. Jones, J. Lau, J. Carpenter, G. Rücker, R.M. Harbord, C.H. Schmid, J. Tetzlaff, J.J. Deeks, J. Peters, P. Macaskill, G. Schwarzer, S. Duval, D.G. Altman, D. Moher, J.P.T. Higgins, Recommendations for examining and interpreting funnel plot asymmetry in meta-analyses of randomised controlled trials. BMJ 343, d4002 (2011). https://doi.org/10.1136/bmj.d4002

26. S. Agarwal, Q. Sadiq, I. Ortanca, Hobnail cells in encapsulated papillary thyroid carcinoma: report of 2 cases with immunohistochemical and molecular findings and literature analysis. Pathol. Res. Pract. 216(3), 152678 (2020). https://doi.org/10.1016/j.prp. 2019.152678

27. S. Asioli, F. Maletta, F. Pagni, D. Pacchioni, A. Vanzati, S. Mariani, N. Palestini, R.V. Lloyd, A. Sapino, Cytomorphologic and molecular features of hobnail variant of papillary thyroid carcinoma: case series and literature review. Diagn. Cytopathol. 42(1), 78-84 (2014). https://doi.org/10.1002/dc.23028

28. C. Bellevicine, I. Cozzolino, U. Malapelle, P. Zeppa, G. Troncone, Cytological and molecular features of papillary thyroid carcinoma with prominent hobnail features: a case report. Acta Cytologica 56 (5), 560-564 (2012). https://doi.org/10.1159/000338395

29. R. Brown, Papillary thyroid carcinoma with prominent hobnail features diagnosed preoperatively by fine needle aspiration and demonstrating constitutive activation of mtor signaling pathway: a case report. J. Clin. Exp. Pathol. 04 (2014). https://doi.org/10. 4172/2161-0681.1000152

30. J.M. Cameselle-Teijeiro, I. Rodriguez-Perez, R. Celestino, C. Eloy, M. Piso-Neira, I. Abdulkader-Nallib, P. Soares, M. Sobrinho-Simoes, Hobnail variant of papillary thyroid carcinoma: clinicopathologic and molecular evidence of progression to undifferentiated carcinoma in 2 cases. Am. J. Surg. Pathol. 41(6), 854-860 (2017). https://doi.org/10.1097/pas.0000000000000793

31. Y.S. Lee, Y. Kim, S. Jeon, J.S. Bae, S.L. Jung, C.K. Jung, Cytologic, clinicopathologic, and molecular features of papillary thyroid carcinoma with prominent hobnail features: 10 case reports and systematic literature review. Int. J. Clin. Exp. Pathol. 8(7), 7988-7997 (2015)

32. M.T. Lilo, J.A. Bishop, S.Z. Ali, Hobnail variant of papillary thyroid carcinoma: a case with an unusual presentation. Diagn. Cytopathol. 45(8), 754-756 (2017). https://doi.org/10.1002/dc.23723

33. S. Mehrotra, R. Lapadat, G.A. Barkan, S.E. Pambuccian, "Teardrop," "comet," and "bowling-pin" cells in a hobnail variant of papillary thyroid carcinoma fine needle aspirate. Diagn. Cytopathol. 47(8), 839-842 (2019). https://doi.org/10.1002/dc.24189

34. L. Teng, W. Deng, J. Lu, J. Zhang, X. Ren, H. Duan, S. Chuai, F. Duan, W. Gao, T. Lu, H. Wu, Z. Liang, Hobnail variant of papillary thyroid carcinoma: molecular profiling and comparison to classical papillary thyroid carcinoma, poorly differentiated thyroid carcinoma and anaplastic thyroid carcinoma. Oncotarget 8(13), 22023-22033 (2017). https://doi.org/10.18632/oncotarget.15786

35. K.S. Wong, T.Y. Chen, S.E. Higgins, B.E. Howitt, J.H. Lorch, E. K. Alexander, E. Marqusee, N.L. Cho, M.A. Nehs, G.M. Doherty, J.A. Barletta, A potential diagnostic pitfall for hobnail variant of papillary thyroid carcinoma. Histopathology (2019). https://doi. org/10.1111/his.14042
36. M.-J. Schlumberger, S. Filetti, I.D. Hay, CHAPTER 14 - Nontoxic Diffuse and Nodular Goiter and Thyroid Neoplasia. In: Melmed, S., Polonsky, K. S., Larsen, P. R., Kronenberg, H. M. (eds.) Williams Textbook of Endocrinology (Twelfth Edition). pp. 440-475. W.B. Saunders, Philadelphia (2011)

37. L. Davies, H.G. Welch, Current thyroid cancer trends in the United States. JAMA Otolaryngol. Head Neck Surg. 140(4), 317-322 (2014). https://doi.org/10.1001/jamaoto.2014.1

38. I.D. Hay, E.J. Bergstralh, J.R. Goellner, J.R. Ebersold, C.S. Grant, Predicting outcome in papillary thyroid carcinoma: development of a reliable prognostic scoring system in a cohort of 1779 patients surgically treated at one institution during 1940 through 1989. Surgery 114(6), 1050-1057 (1993). Discussion 1057-1058

39. Y. Ito, A. Miyauchi, M. Kihara, M. Fukushima, T. Higashiyama, A. Miya, Overall survival of papillary thyroid carcinoma patients: a single-institution long-term follow-up of 5897 patients. World J. Surg. 42(3), 615-622 (2018). https://doi.org/10.1007/s00268-0184479-z

40. I.D. Hay, G.B. Thompson, C.S. Grant, E.J. Bergstralh, C.E. Dvorak, C.A. Gorman, M.S. Maurer, B. McIver, B.P. Mullan, A. L. Oberg, C.C. Powell, J.A. van Heerden, J.R. Goellner, Papillary thyroid carcinoma managed at the Mayo Clinic during six decades (1940-1999): temporal trends in initial therapy and long-term outcome in 2444 consecutively treated patients. World J. Surg. 26 (8), 879-885 (2002). https://doi.org/10.1007/s00268-002-6612-1

41. M. Xing, A.S. Alzahrani, K.A. Carson, D. Viola, R. Elisei, B. Bendlova, L. Yip, C. Mian, F. Vianello, R.M. Tuttle, E. Robenshtok, J.A. Fagin, E. Puxeddu, L. Fugazzola, A. Czarniecka, B. Jarzab, C.J. O’Neill, M.S. Sywak, A.K. Lam, G. Riesco-Eizaguirre, P. Santisteban, H. Nakayama, R.P. Tufano, S.I. Pai, M.A. Zeiger, W.H. Westra, D.P. Clark, R. Clifton-Bligh, D. Sidransky, P.W. Ladenson, V. Sykorova, Association between BRAF V600E mutation and mortality in patients with papillary thyroid cancer. JAMA 309(14), 1493-1501 (2013). https://doi.org/10.1001/jama.2013.3190

42. A. Czarniecka, M. Oczko-Wojciechowska, M. Barczyński, BRAF V600E mutation in prognostication of papillary thyroid cancer (PTC) recurrence. Gland. Surg. 5(5), 495-505 (2016). https://doi. org/10.21037/gs.2016.09.09

43. R. Elisei, C. Ugolini, D. Viola, C. Lupi, A. Biagini, R. Giannini, C. Romei, P. Miccoli, A. Pinchera, F. Basolo, BRAF(V600E) mutation and outcome of patients with papillary thyroid carcinoma: a 15year median follow-up study. J. Clin. Endocrinol. Metab. 93(10), 3943-3949 (2008). https://doi.org/10.1210/jc.2008-0607

44. G.S. Falchook, M. Millward, D. Hong, A. Naing, S. Piha-Paul, S. G. Waguespack, M.E. Cabanillas, S.I. Sherman, B. Ma, M. Curtis, V. Goodman, R. Kurzrock, BRAF inhibitor dabrafenib in patients with metastatic BRAF-mutant thyroid cancer. Thyroid 25(1), 71-77 (2015). https://doi.org/10.1089/thy.2014.0123

45. K.B. Kim, M.E. Cabanillas, A.J. Lazar, M.D. Williams, D.L. Sanders, J.L. Ilagan, K. Nolop, R.J. Lee, S.I. Sherman, Clinical responses to vemurafenib in patients with metastatic papillary thyroid cancer harboring BRAF(V600E) mutation. Thyroid 23 (10), 1277-1283 (2013). https://doi.org/10.1089/thy.2013.0057

46. V. Subbiah, R.J. Kreitman, Z.A. Wainberg, J.Y. Cho, J.H.M. Schellens, J.C. Soria, P.Y. Wen, C. Zielinski, M.E. Cabanillas, G. Urbanowitz, B. Mookerjee, D. Wang, F. Rangwala, B. Keam, Dabrafenib and trametinib treatment in patients with locally advanced or metastatic BRAF V600-mutant anaplastic thyroid cancer. J. Clin. Oncol. 36(1), 7-13 (2017). https://doi.org/10. 1200/JCO.2017.73.6785

47. L.A. Dunn, E.J. Sherman, S.S. Baxi, V. Tchekmedyian, R.K. Grewal, S.M. Larson, K.S. Pentlow, S. Haque, R.M. Tuttle, M.M. Sabra, S. Fish, L. Boucai, J. Walters, R.A. Ghossein, V.E. Seshan, A. Ni, D. Li, J.A. Knauf, D.G. Pfister, J.A. Fagin, A.L. Ho, Vemurafenib redifferentiation of BRAF mutant, RAI-refractory 
thyroid cancers. J. Clin. Endocrinol. Metab. 104(5), 1417-1428 (2019). https://doi.org/10.1210/jc.2018-01478

48. M.J. Schlumberger, Papillary and follicular thyroid carcinoma. N. Engl. J. Med. 338(5), 297-306 (1998). https://doi.org/10.1056/ nejm199801293380506

49. J. Hoie, A.E. Stenwig, G. Kullmann, M. Lindegaard, Distant metastases in papillary thyroid cancer. A review of 91 patients.
Cancer 61(1), 1-6 (1988). https://doi.org/10.1002/1097-0142 (19880101)61:1<1::aid-cncr2820610102>3.0.co;2-r

50. Y. Liu, Y. Wang, K. Zhao, D. Li, Z. Chen, R. Jiang, X. Wang, X. $\mathrm{He}$, Lymph node metastasis in young and middle-aged papillary thyroid carcinoma patients: a SEER-based cohort study. BMC Cancer 20(1), 181 (2020). https://doi.org/10.1186/s12885-020$6675-0$ 